\title{
Mortalidade por desastres no Brasil: o que mostram os dados
}

\author{
Mortality due to disasters in Brazil: what the data reveals
}

Roberto Luiz do Carmo ${ }^{1}$

Tathiane Mayumi Anazawa ${ }^{1}$

${ }^{1}$ Núcleo de Estudos de População, Universidade Estadual de Campinas. R. Albert Einstein 1300, Cidade Universitária. 13.083-852 Campinas SP Brasil. roberto@nepo.unicamp.br
Abstract This work presents and analyzes the main databases on mortality due to disasters in Brazil: EM-DAT - Emergency Events Database and the Brazilian Atlas of Natural Disasters, as well as the Mortality Information System (SIM, Ministry of Health) and the Yearbook of Natural Disasters (Ministry of National Integration). These databases were addressed using two basic methodological procedures: descriptive analysis of systematic information and comparative analysis, by means of the construction of tables that helped to analyze the information selected. The comparison revealed that with the current databases it is not possible to affirm if disasters and mortality due to disasters are increasing in Brazil, since there are variations in the intensity of the events that occur each year. The information available shows the importance of the mega disaster that occurred in the mountainous region of Rio de Janeiro in 2011, especially in the municipality of Nova Friburgo. In this case the disaster affected the various age groups in both sexes in different ways: the 5 to 9-year-old age group was the most affected among men and women, as well as the 20 to 24-year-old age group (for women) and the 30 to 34-year-old age group (for men).

Key words Disasters, Mortality, Database, Mega disaster
Resumo Este trabalho tem como objetivo apresentar e analisar as principais bases de dados existentes sobre mortalidade por desastres ocorridos no Brasil: EM-DAT - Emergency Events Database $e$ o Atlas Brasileiro de Desastres Naturais, além do Sistema de Informações sobre Mortalidade (SIM, Ministério da Saúde) e o Anuário de Desastres Naturais (Ministério da Integração Nacional). Essas bases de dados foram abordadas através de dois procedimentos metodológicos básicos: análise descritiva das informações sistematizadas e análise comparativa, através da construção de tabelas que permitiram analisar informações selecionadas. A comparação mostrou que, com as bases de dados atuais, não é possível afirmar se os desastres e a mortalidade por desastres estão aumentando no país, por conta das variações dos eventos que ocorrem a cada ano. O levantamento das informações mostrou a importância do megadesastre ocorrido na Região Serrana do Rio de Janeiro em 2011, em especial no município de Nova Friburgo. Neste municipio, o desastre atingiu de diferentes formas os diversos grupos etários em ambos os sexos: o de 5 a 9 anos, foi o mais atingido entre homens e mulheres, além dos de 20 a 24 anos (para mulheres) e 30 a 34 (para homens).

Palavras-chave Desastres, Mortalidade, Base de dados, Megadesastre 


\section{Introdução}

Os eventos climáticos, associados às características assumidas pelas formas de ocupação do espaço pela população, historicamente foram responsáveis pela ocorrência de desastres, provocando um número considerável de perdas de vidas humanas a cada ano, além de perdas materiais expressivas. No contexto em que se discute o acirramento das mudanças ambientais globais, com o aumento dos eventos climáticos extremos ${ }^{1}$, essa questão se torna cada vez mais relevante.

Este trabalho apresenta uma análise das fontes de dados sobre mortalidade por desastres ocorridos no Brasil, indicando as suas potencialidades e as lacunas ainda existentes. As bases de dados sobre desastres analisadas são as mais abrangentes e detalhadas disponíveis para consulta pública: uma base de dados de escala global (EM-DAT - Emergency Events Database) e outra de escala nacional (Atlas Brasileiro de Desastres Naturais). Estas bases de dados foram abordadas através de dois procedimentos metodológicos básicos: análise descritiva das informações sistematizadas e análise comparativa, através da construção de tabelas que permitiram o emparelhamento das informações selecionadas. Além do número de óbitos, foram trabalhadas também as informações referentes às características da população vitimada por desastres, em termos de sua composição por sexo e idade, analisando a potencialidade dos dados do Sistema de Informação sobre Mortalidade (SIM). O levantamento dessas informações demonstrou as proporções impressionantes dos óbitos decorrentes do desastre na Região Serrana do Rio de Janeiro, no ano 2011. Por conta disso, esse caso foi considerado de maneira detalhada.

Nesse sentido, o presente trabalho objetiva responder às questões: 1) O que evidenciam as bases de dados disponíveis sobre a caracterização e impactos dos desastres? 2) as bases de dados existentes permitem identificar se a mortalidade decorrente de desastres está aumentando? 3) É possível observar um padrão de mortalidade para os municípios mais atingidos por desastres?

Considerando essas questões, as hipóteses levantadas são respectivamente: a) cada uma das bases de dados apresenta resultados específicos, com acuidade diferenciada; b) as bases de dados devem mostrar que a mortalidade por causas relacionadas a desastres está aumentando; c) a mortalidade por desastres é diferencial por sexo e por idade, sendo que a composição etária de uma população pode influenciar na vulnerabilidade aos desastres.

\section{Desastres como construção social}

Os desastres são amplamente discutidos pela literatura desde o início do Séc. XX, conforme apresentado por Dynes e Drabek ${ }^{2}$, e também por Quarantelli e Dynes ${ }^{3}$. A perspectiva que adotamos neste trabalho é de que os desastres, situações extremas nas quais a vida e os bens materiais são ameaçados, são uma construção social. Especificamente no caso do Brasil, os diversos desastres registrados recentemente decorrem da forma de ocupação do espaço, que desconsidera, principalmente por motivos econômicos, os riscos representados, por exemplo, pela declividade acentuada do terreno e a proximidade de cursos d'água.

As mudanças climáticas, em suas conexões com as mudanças ambientais globais, evidenciam a relevância de um "novo" conjunto de agentes físicos capazes de potencializar as situações de desastre, considerando a previsão de que os eventos climáticos extremos devem aumentar em número e intensidade ${ }^{1}$. No caso brasileiro, a discussão sobre as decorrências das mudanças climáticas, seja através da abordagem dos riscos ou da abordagem dos perigos, desenvolve-se em um contexto de transições importantes: a transição demográfica e a transição urbana.

A transição demográfica tem sido descrita como o processo através do qual a população de um determinado país passa de níveis altos de natalidade e mortalidade para níveis mais baixos de natalidade e mortalidade. Como no processo a mortalidade decresce em um momento anterior à natalidade, ocorre um crescimento significativo antes da diminuição dos respectivos níveis. A transição demográfica é amplamente discutida na literatura demográfica ${ }^{4-6}$. Esse processo tem como resultado a diminuição das taxas de crescimento e o envelhecimento relativo da população, com o aumento do peso relativo dos idosos em função da redução das taxas de fecundidade ${ }^{5}$. Essas mudanças, que se configuram na estrutura etária, são muito importantes para ajudar a compreender as necessidades das populações potencialmente expostas a desastres, assim como para possibilitar a construção de planos de enfrentamento dos desastres que sejam mais efetivos.

Durante o Século XX, o Brasil passou por uma transformação importantíssima em termos da redistribuição espacial de sua população. $\mathrm{O}$ país, que era predominantemente rural na década de 1950 (36\% da população residindo em áreas rurais), passou por uma grande concentração populacional nas áreas definidas como urbanas, chegando ao ano de 2010 com $84 \%$ da população 
urbana. Entretanto, esse processo não foi acompanhado pelos investimentos necessários em termos de infraestrutura e de serviços públicos nas cidades, o que fez com que a transição urbana no Brasil seja caracterizada como um processo incompleto, que reproduz na ocupação do espaço urbano as características da desigualdade social ${ }^{6}$. Desse processo resultou a concentração da população de baixa renda em determinados espaços, nem sempre com características geomorfológica ou de localização adequadas à ocupação humana. Essas populações, residindo em ocupações inadequadas, são as principais vítimas das situações de desastre ${ }^{7}$.

Nesse contexto, é fundamental o registro e a análise das consequências dos desastres, de maneira a subsidiar políticas públicas capazes de reduzir as perdas, especialmente de vidas humanas. Considerando estes aspectos, o objetivo deste trabalho é apresentar e discutir os dados sobre mortalidade decorrente de desastres no Brasil, atentando especialmente para as fontes de dados e suas características, apresentadas a seguir.

\section{O que evidenciam as bases de dados disponíveis sobre a caracterização e impactos dos desastres?}

A busca por dados relativos a desastres, seja em termos de suas causas, seja em termos de suas consequências, tornou-se fundamental no período recente. Os tomadores de decisão estão utilizando cada vez mais esses dados, tanto para medidas mitigadoras, quanto para o monitoramento das áreas afetadas.

Como base de dados consolidada, cita-se o EM-DAT (Emergency Events Database), que consiste em um banco de dados internacional sobre desastres. O EM-DAT foi desenvolvido e é administrado pelo Centre for Research on the Epidemiology of Disasters (CRED) da Universidade de Louvain (Bélgica), com suporte da Office of Foreign Disaster Assistance (OFDA).

Esta base possui dados de desastres contabilizados a partir de 1900 e sua construção é realizada a partir de diversas fontes, como as agências das Organização das Nações Unidas (ONU), organizações não governamentais, companhias de seguro, instituições de pesquisa e agências de notícias ${ }^{8}$. O principal objetivo da base de dados é servir aos propósitos da ação humanitária em nível nacional e internacional.

O EM-DAT utiliza alguns critérios para que a "ocorrência de desastre" seja computada: $10 \mathrm{ou}$ mais óbitos; 100 ou mais pessoas afetadas; decla- ração de estado de emergência e pedido de auxílio internacional.

A vantagem notória do EM-DAT deve-se ao fato de sua abrangência internacional, resultando em uma padronização nos conceitos relacionados aos desastres. Esta base de dados, apesar de sua abrangência de âmbito internacional, fácil acesso aos dados e disponibilização gratuita, apresenta algumas limitações quanto a suas estimativas de vítimas de desastres, como será evidenciado mais adiante. O EM-DAT também se destaca por seu uso frequente pela ONU, para nortear suas ações e políticas relacionadas aos desastres ${ }^{8}$.

Em relação ao registro dos desastres no Brasil, o Anuário de Desastres Naturais ${ }^{9}$ destaca três iniciativas da Secretaria Nacional de Defesa Civil, em 2012, que auxiliaram o conhecimento do histórico e perfil dos desastres no Brasil: (1) Atlas Brasileiro de Desastres Naturais 1991 a 2010, organizado pelo Centro Universitário de Estudos e Pesquisas sobre Desastres (CEPED), da Universidade Federal de Santa Catarina - UFSC; (2) Diagnóstico do Plano Nacional de Gestão de Riscos e Resposta a Desastres (PNGRD), organizado pela Fundação Getúlio Vargas (FGV) e; (3) Sistema Integrado de Informações sobre Desastres (S2ID). Neste trabalho destaca-se o Atlas Brasileiro de Desastres Naturais.

O Atlas Brasileiro de Desastres Naturais é resultado de uma pesquisa realizada pelo Centro Universitário de Estudos e Pesquisas sobre Desastres (CEPED) da Universidade Federal de Santa Catarina, com cooperação da Secretaria Nacional de Defesa Civil. Essa pesquisa teve como objetivos a compilação e disponibilização de informações sobre os registros de desastres ocorridos no Brasil no período de 1991 a 2010, publicados em 26 Volumes Estaduais e um Volume Brasil ${ }^{10}$. Essa pesquisa consiste em um marco no âmbito nacional, pois até então não havia uma compilação dos dados relacionados a desastres em todo o território brasileiro, com informações específicas e organizadas, enfatizando a análise temporal e espacial dos desastres. Segundo o $\operatorname{CEPED}^{10}$, a importância do Atlas deve-se a contextualização histórica dos desastres, construindo um perfil das ocorrências dos desastres no Brasil e as especificidades por Regiões e por Unidades da Federação. Esta pesquisa fornece subsídios para o planejamento adequado em gestão de risco e redução de desastres.

Por outro lado, não há informações detalhadas por ano de ocorrência, faixa etária e sexo, que forneceria aos pesquisadores um instrumental analítico mais robusto. 
Para caracterizar as decorrências dos desastres, foram sistematizados os óbitos contabilizados no EM-DAT e no Atlas Brasileiro de Desastres Naturais. Deve-se enfatizar a comparabilidade dos bancos de dados, uma vez que no Atlas são registradas apenas as ocorrências que levaram os municípios a decretarem Situação de Emergência (SE) ou Estado de Calamidade Pública (ECP), os quais são compatíveis com os critérios do EM-DAT.

Analisando as bases de dados, no período de 1991-2010 (Tabela 1), é possível observar que há discrepâncias tanto no número total de ocorrências de desastres, quanto no número de óbitos e pessoas afetadas, que se referem ao número de pessoas vitimadas, de alguma forma, em consequência do desastre. Foram computados 103 eventos na base do EM-DAT, um número pouco expressivo quando comparado ao total do Atlas, de 31.909 registros de desastres no mesmo período. Estes eventos seguem os critérios de registro do EM-DAT, mas a maior parte deles não foi inserida na base, resultando em um grave erro de omissão.

Os bancos do EM-DAT e do Atlas apresentam resultados similares em relação ao aumento dos desastres nos últimos anos. Porém, algumas diferenças podem ser observadas em relação aos tipos de desastres. A diferença mais acentuada está entre as secas e estiagens. No Atlas, os desastres com maior registro são as secas e estiagem (53,41\%), para o período de 1991-2010. Já para o EM-DAT, as secas correspondem apenas a 7,77\% do total dos registros. Para o EM-DAT, as inundações foram os tipos de desastre mais frequente, representando 44,66\% do total. Já para o Atlas, as inundações bruscas e graduais totalizam 32,92\% do total de desastres registrados para o período considerado. Outra diferença observada está em relação aos eventos de movimento de massa $(12,62 \%$ do total de registros, no EM-DAT, e, $1,43 \%$, no Atlas).

Devido às diferentes definições e metodologias de coleta de dados entre as fontes analisadas,

Tabela 1. Total de registro de desastres, óbitos e pessoas afetadas por desastres no Brasil no período de 1991 a 2010.

\begin{tabular}{lrrr}
\hline \multicolumn{1}{c}{$\begin{array}{c}\text { Fonte } \\
\text { de dados }\end{array}$} & $\begin{array}{c}\text { Registro de } \\
\text { desastre }\end{array}$ & Óbitos & $\begin{array}{c}\text { Pessoas } \\
\text { afetadas }\end{array}$ \\
\hline EM-DAT & 103 & 2.689 & 18.266 .784 \\
Atlas Brasileiro & 31.909 & 3.404 & 96.220 .879 \\
\hline
\end{tabular}

Fonte: EM-DAT e CEPED. não há um consenso entre os valores apresentados. O EM-DAT, por coletar dados a partir de determinados critérios citados acima, omitiu 13\% dos valores totais de óbitos em relação ao Atlas Brasileiro de Desastres Naturais. O Atlas apresentou maior cobertura em todos os quesitos, principalmente no número de registro de desastres, apresentando maior detalhamento dos eventos ocorridos em todo o território nacional, a partir da coleta de dados. Esses dados foram obtidos junto às Coordenadorias Estaduais de Defesa Civil, a partir de documentos oficiais de registros de desastres disponibilizados. Foram considerados como: (a) relatório de danos; (b) AVADAN (Avaliação de Danos); (c) NOPRED (Notificação Preliminar de Desastre); (d) Decretos; e (e) Portarias.

Os dados do EM-DAT para o Brasil são muito menores quando se faz uma comparação com o Atlas, em números de registro de desastre, de óbitos e de pessoas afetadas. Este fato também foi observado por Marcelino et al. ${ }^{11}$, que analisaram os dados do EM-DAT e do Departamento Estadual de Defesa Civil (DEDC-SC). Segundo os autores, foram computados no EM-DAT 89 eventos de desastres no período de 1980-2003, para o Brasil, enquanto o DEDC-SC registrou 3.373 eventos de desastres, somente no Estado de Santa Catarina. De acordo com Marcelino et al. ${ }^{11}$ os registros dos desastres apresentam erros de classificação no AVADAN, enviado à Defesa Civil. Além disso, há problemas na superestimação dos danos causados pelos desastres, que pode se configurar em uma tentativa dos governos municipais de obter recursos externos. Além disso, são citadas as limitações como a inconsistência no preenchimento dos relatórios, como por exemplo, os dados de danos humanos, materiais e econômicos.

Outra fonte de dados sobre a mortalidade decorrente dos desastres consiste no Sistema de Informações sobre Mortalidade (SIM), criado pelo Ministério da Saúde em 1975, informatizado em 1979, que, com a criação do Sistema Único de Saúde (SUS), sua manutenção foi direcionada ao Departamento de Informática do SUS (DATASUS). O SIM apresenta objetivo de reunir dados qualitativos e quantitativos sobre os óbitos ocorridos no Brasil. O potencial desta fonte de dados é baseado na obtenção regular dos dados de mortalidade, a partir da declaração de óbito (DO), do país e sua caracterização e detalhamento ${ }^{12}$.

No SIM, a causa básica de óbito é codificada a partir do declarado pelo médico atestante, segundo regras estabelecidas pela Organização Mundial de Saúde. A partir de 1996, as declarações de óbito passaram a ser codificadas uti- 
lizando-se a 10ª Revisão da Classificação Internacional de Doenças - CID-10 ${ }^{13}$. Na CID-10, um agrupamento inserido no capítulo XX - Causas Externas de Morbidade e Mortalidade refere-se aos desastres sobre os quais nos detivemos neste trabalho: o "Agrupamento (X30-X39) - Exposição às Forças da Natureza”.

A partir de informações de causa básica de óbitos, há a possibilidade de se obter informações detalhadas sobre óbitos decorrentes de determinada causa de morte, como óbitos por faixas etárias, por sexo, por município, por ano, entre outras informações. A cobertura do SIM tem avançado de maneira significativa ao longo do tempo, entretanto, persiste a crítica no que diz respeito à classificação errônea dos óbitos, com o preenchimento equivocado ou incompleto do formulário de óbito.

Foi realizada outra comparação entre os Anuários de Desastres Naturais e o SIM (utilizando dados referentes ao Agrupamento X30-X39 Exposição às Forças da Natureza), para o ano de 2011, para o qual havia registros nas duas bases. Observa-se na Tabela 2 que o valor de óbitos no SIM (1042 óbitos), relativos ao Brasil, é menor que o valor registrado no Anuário de Desastres Naturais (1094 óbitos). Porém, a diferença é relativamente pequena, 52 casos (menos de $5 \%$ ). O menor valor registrado pelo SIM pode ser reflexo de uma classificação equivocada da causa básica de óbito. Existe a possibilidade do desastre também não ser a causa básica de óbitos, apresentando outras causas, consequentes ao desastre, como causa básica de óbito.

O SIM e o Anuário de Desastres Naturais revelaram que o ano de 2011 concentrou o maior volume de óbitos decorrentes dos desastres (Tabela 3). O Anuário de Desastres Naturais de $2012^{14}$ registrou para o ano de 2012 um total de 93 óbitos. O grande volume de óbitos em 2011 deve-se ao megadesastre da região Serrana do Rio de Janeiro, que deixou 912 mortos, além de 350 pessoas desaparecidas e 45 mil pessoas desabrigadas. Este evento foi considerado um megadesastre, o maior da história do país ${ }^{14}$. Já em 2012, o número de afetados foi maior devido aos resultados das secas/estiagem na região Nordeste.

Os diferentes totais apresentados pelas bases de dados disponíveis evidencia que ainda é necessário investir na criação de um escopo comum, que seja capaz de identificar e registrar as informações de maneira precisa, e com isso servir como subsídio para políticas públicas mais efetivas.
Tabela 2. Total de registro de óbitos decorrentes de desastre no Brasil em 2011.

\begin{tabular}{lc}
\hline \multicolumn{1}{c}{ Fonte de dados } & Total de óbitos em 2011 \\
\hline SIM & 1.042 \\
Anuário de Desastres Naturais & 1.094 \\
\hline
\end{tabular}

Fonte: Sistema de Informação sobre Mortalidade (SIM/ DATASUS) - Agrupamento X30-X39 - Exposição às forças da natureza e Anuário de Desastres Naturais 2011.

Tabela 3. Total de registro de desastres, óbitos e danos humanos por desastres no Brasil nos anos de $2011 \mathrm{e}$ 2012.

\begin{tabular}{lrr}
\hline & \multicolumn{2}{c}{ Ano } \\
\cline { 2 - 3 } & $\mathbf{2 0 1 1}$ & $\mathbf{2 0 1 2}$ \\
\hline Registros & 795 & 376 \\
Óbitos & 1.094 & 93 \\
Danos Humanos (indivíduos) & 12.535 .401 & 16.977 .614 \\
\hline
\end{tabular}

Fonte: Anuário de Desastres Naturais (2011 e 2012).

\section{As bases de dados existentes permitem identificar se a mortalidade decorrente de desastres está aumentando?}

A intensificação da frequência dos desastres no mundo tem início na década de 1950, agravando-se nas décadas de 1980 e 2000. Estas observações indicam não só a elevação da frequência dos desastres, mas também a intensificação dos eventos extremos, e suas consequências para a sociedade. De acordo com os dados obtidos junto ao EM-DAT, na década de 1970, a média de desastres foi de 90 eventos por ano. Já na década de 1990, expressivos 300 eventos por ano passaram a compor a média neste período, saltando para 450 desastres por ano na década de 2000 (Figura 1).

Segundo o EM-DAT, no período de 1991 a 2010, foram registrados no mundo 7.602 desastres, totalizando 1.620.093 óbitos e 4.496.909.737 pessoas afetadas ${ }^{4}$. Ao analisar os dados para o período considerado, observa-se um aumento na frequência de ocorrência dos desastres, enfatizando os anos de 2000 e 2010, que registraram 526 e 533 desastres, respectivamente, os maiores registros encontrados para o período.

O Atlas Brasileiro de Desastres Naturais indica que houve um aumento expressivo no núme- 


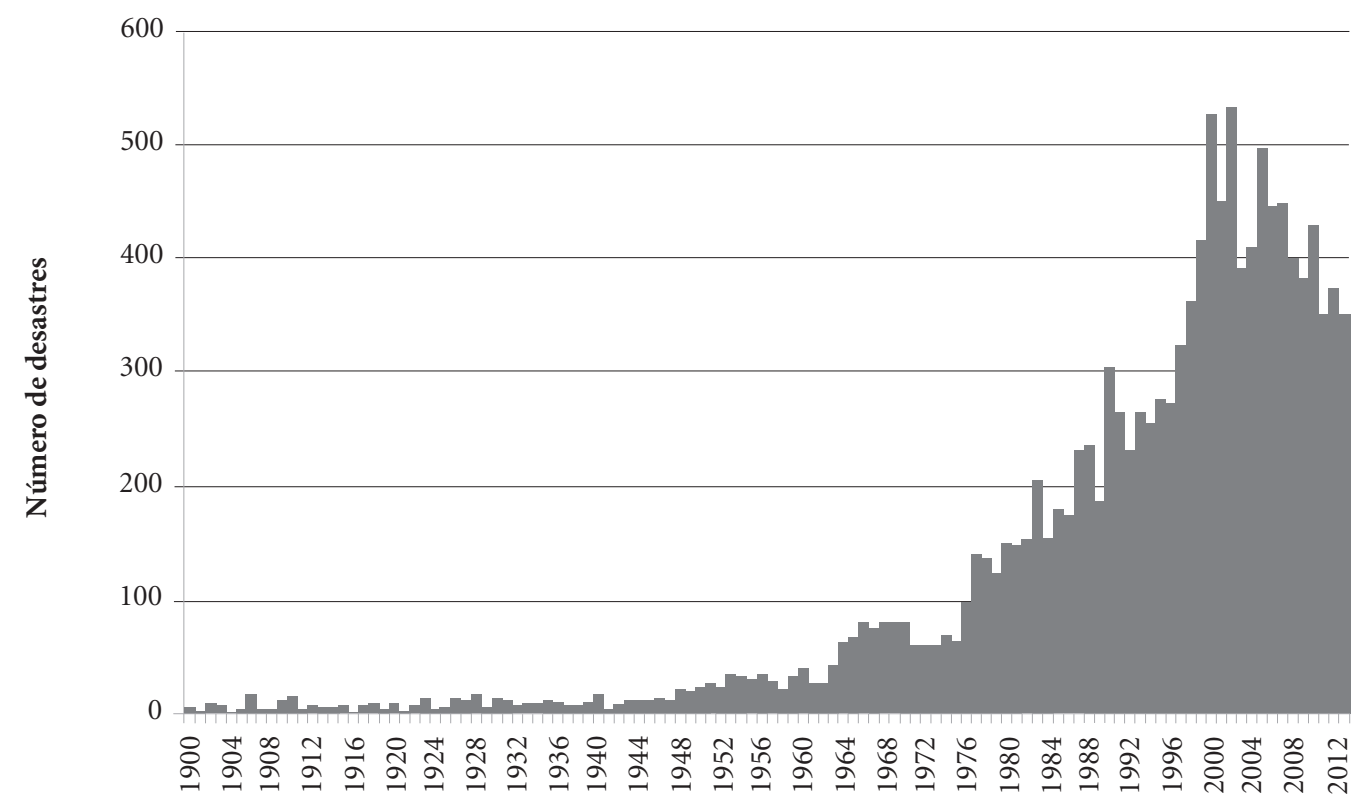

Ano

Figura 1. Registros de desastres no mundo, no período de 1900 a 2013. Fonte: EM-DAT.

ro de ocorrências de desastres, totalizando 8.671 ocorrências na década de 1990, e 23.238 na década de 2000. No entanto, o CEPED alerta sobre o cuidado ao afirmar que os desastres aumentaram em 73\% nos últimos 10 anos. Há que se considerar o sistema de atualização e registro do Sistema de Defesa Civil ${ }^{15}$.

Ao discutir os desastres sob a ótica dos seus extremos, podemos considerar as enchentes e a estiagem e seca. Ao observar os 10 maiores desastres ocorridos no Brasil, segundo o Em-DAT, considerando o número de pessoas afetadas, os quatro principais estão relacionados à seca, ocorridos em setembro de 1983 (20.000.000 pessoas afetadas), agosto de 1970 e abril de 1998 (10.000.000 pessoas afetadas em cada evento) e em 1979 (5.000.000 pessoas afetadas). As inundações também aparecem como desastres que afetaram as pessoas, mas com menor intensidade em relação à seca. Estes eventos ocorreram em fevereiro de 1988 e janeiro de 1983 (cerca de 3.000.000 pessoas afetadas em cada evento), em novembro de 2008 e janeiro de 1979 (cerca de 1.500.000 pessoas afetadas em cada evento) e em abril de 2009 (1.150.900 pessoas afetadas) ${ }^{8}$.

A distribuição dos danos humanos para o período considerado (1991-2010) aponta que a estiagem e seca são os desastres que mais afetam pessoas $(53,41 \%)$, por serem mais recorrentes. Já as inundações bruscas atingem $21,34 \%$ da população do Brasil e causam maior número de óbitos $(43,19 \%)$, enquanto que os óbitos por seca e estiagem representam $10,38 \%{ }^{10}$.

Quando comparada por regiões, a distribuição dos danos humanos concentra-se na região Nordeste, com ênfase nos ocasionados pela seca, com cerca de 59\% dos danos humanos do total brasileiro. Em seguida, o Sul apresenta 25\% dos danos humanos ocasionados pela seca, porém, o desastre que mais afetou pessoas na região Sul, foi a inundação brusca. Já no Sudeste, percebese que as inundações bruscas e graduais são os desastres que ocasionam os maiores danos na região. As regiões Centro-Oeste e Norte apresentaram os menores valores de danos humanos ocasionados por desastres ${ }^{10}$.

A região Sudeste apresenta grande parte das ocorrências de estiagem e secas (35\%) e inundações bruscas $(32 \%)$ do total registrado para o país ${ }^{10}$. A diversidade de regimes climáticos da região é decorrente da variabilidade longitudinal e de relevo, a maritimidade e continentalidade, além da atuação de sistemas tropicais e extratropicais de latitudes médias ${ }^{10}$. 
Para analisar os desastres ocorridos especialmente no estado do Rio de Janeiro, adotamos como base de dados para responder a esta questão o Sistema de Informação de Mortalidade (SIM) mesmo considerando as discrepâncias que foram apontadas no item anterior e tendo em vista que esta é a função precípua deste sistema. A suposição é que intrinsecamente as variações são pouco significativas ao longo do tempo, em termos de conceitos e forma de obtenção da informação.

A partir de dados do SIM, mostrados na Tabela 4, observa-se que o Estado do Rio de Janeiro se destaca das demais Unidades de Federação (UFs), principalmente no ano de 2010, com 264 óbitos devido à avalanche, desabamento de terra e outros movimentos da superfície terrestre (CID-10: X36). Em um período de 13 anos (1996-2009), a média de óbitos no Estado do Rio de Janeiro foi de 19 óbitos devido a esta causa básica. Em 2010, o número de óbitos foi de 264, refletindo um peso significativo dos desastres sobre a mortalidade do Estado.

Assim como as vítimas de avalanche, desabamento de terra e outros movimentos da superfície terrestre, o volume de óbitos classificados como vítimas de tempestade cataclísmica (Categoria CID-10: X37) no Estado do Rio de Janeiro foi grande em 2011, totalizando 858 óbitos, segundo o SIM. Esse foi um número extremamente elevado quando comparado à média em 15 anos (1996-2010), de 5 óbitos no Estado do Rio de Janeiro, por ano (Tabela 4). Este valor, que impacta o total de óbitos por desastres no Brasil, está relacionado com o megadesastre da Região Serrana, ocorrido em 2011.

O que os dados permitem concluir é que não existe uma tendência clara de aumento do número de óbitos associados às causas de morte relacionadas a desastres ao longo do período considerado (1996 a 2011). Existem flutuações que são significativas a cada ano, que poderiam ser ainda maiores se fossem considerados os episódios de desastres ocorridos em décadas anteriores.

O Megadesastre da Região Serrana totalizou 912 óbitos, e atingiu com maior intensidade os municípios de Nova Friburgo (429 óbitos), Teresópolis (388 óbitos) e Petrópolis (71 óbitos) ${ }^{15}$. Historicamente, estes municípios já foram palco de outros desastres, como deslizamentos em 1987 em Petrópolis e Teresópolis, com 282 óbitos. Em 1988 , Petrópolis passou pela pior enchente, contabilizando 277 mortos e dois mil desabrigados. Nova Friburgo, Petrópolis e Teresópolis foram devastados pela chuva, resultando em 5 óbitos.

Tabela 4. Total de óbitos no Estado do Rio de Janeiro, demais Unidades de Federação e no Brasil, no período de 1996-2011. Categoria CID-10: X36 - Vítima de avalanche, desabamento de terra e outros movimentos da superfície terrestre e Categoria CID-10: X37 - Vítima de tempestade cataclísmica.

\begin{tabular}{|c|c|c|c|c|c|c|}
\hline & \multicolumn{2}{|c|}{ Rio de Janeiro } & \multicolumn{2}{|c|}{ Demais UFs } & \multicolumn{2}{|c|}{ Brasil } \\
\hline & $\begin{array}{c}\text { Categoria } \\
\text { CID-10: X36 }\end{array}$ & $\begin{array}{r}\text { Categoria } \\
\text { CID-10: X37 }\end{array}$ & $\begin{array}{r}\text { Categoria } \\
\text { CID-10: X36 }\end{array}$ & $\begin{array}{r}\text { Categoria } \\
\text { CID-10: X37 }\end{array}$ & $\begin{array}{r}\text { Categoria } \\
\text { CID-10: X36 }\end{array}$ & $\begin{array}{l}\text { Categoria } \\
\text { CID-10: X37 }\end{array}$ \\
\hline 1996 & 43 & 1 & 64 & 2 & 107 & 3 \\
\hline 1997 & 2 & 1 & 10 & 9 & 12 & 10 \\
\hline 1998 & 3 & 1 & 9 & 14 & 12 & 15 \\
\hline 1999 & 6 & - & 24 & 9 & 30 & 9 \\
\hline 2000 & 14 & 1 & 27 & 2 & 41 & 3 \\
\hline 2001 & 4 & - & 25 & 11 & 29 & 11 \\
\hline 2002 & 43 & 12 & 27 & 8 & 70 & 20 \\
\hline 2003 & 32 & 2 & 33 & 16 & 65 & 18 \\
\hline 2004 & 10 & 8 & 26 & 6 & 36 & 14 \\
\hline 2005 & 11 & 2 & 21 & 3 & 32 & 5 \\
\hline 2006 & 18 & 11 & 34 & 7 & 52 & 18 \\
\hline 2007 & 33 & 4 & 25 & 8 & 58 & 12 \\
\hline 2008 & 15 & 8 & 124 & 2 & 139 & 10 \\
\hline 2009 & 27 & 3 & 34 & 12 & 61 & 15 \\
\hline 2010 & 264 & 4 & 66 & 12 & 330 & 16 \\
\hline 2011 & 27 & 858 & 50 & 14 & 77 & 872 \\
\hline Total & 552 & 916 & 599 & 135 & 1.151 & 1.051 \\
\hline
\end{tabular}

Fonte: Sistema de Informação sobre Mortalidade (SIM/DATASUS). 
Em 2007 foram 10 óbitos em Nova Friburgo, Sumidouro, Petrópolis e Teresópolis. E em 2008, Petrópolis registrou 9 mortes $^{16}$.

Segundo o $\mathrm{IBGE}^{16}$, Teresópolis apresenta 163.746 habitantes em 2010, com 10,71\% (17.539 habitantes) residindo em área rural e 89,29\% (146.207 habitantes) na área urbana. Sua densidade demográfica é de 212,49 habitantes $/ \mathrm{km}^{2}{ }^{16}$. Já Petrópolis, com 295.917 habitantes em 2010, apresenta a menor proporção da população localizada em área rural (4,94\%), totalizando 14.631 habitantes. Em contrapartida, 95,06\% (28.286 habitantes) da população estão concentrados na área urbana. A densidade demográfica do município é de 371,85 habitantes $/ \mathrm{km}^{2}$. Em Nova Friburgo 87,53\% (159.372 habitantes) reside em área urbana e 12,47\% (22.710 habitantes) em área rural, totalizando uma população de 182.082 habitantes em 2010. A densidade demográfica do município é de 195,07 habitantes/ $\mathrm{km}^{2}{ }^{16}$.

Áreas com alta densidade demográfica relacionadas com outros fatores, como ocupação desordenada em área de risco, podem agravar situações de adversidades atmosféricas ${ }^{14}$. Contudo, esta situação não foi observada na região Serrana do Rio de Janeiro. As fortes chuvas ocorreram de forma generalizada nesta região, porém, sua forma de deslocamento, a quantidade e as características geomorfológicas de cada bacia hidrográfica da região Serrana do Rio de Janeiro, causaram impactos com intensidades e formatos diferenciados, além de diversos movimentos em massa (tipo Parroca, Rasteira, Vale Suspenso e Catarina) em cada município ${ }^{17}$. Apesar da diversificação dos movimentos de massa e a intensidade de cada ocorrência, houve uma devastação de maneira igualitária nas áreas rurais e urbanas, moradores de baixa e alta renda, bem como áreas agrícolas, infraestruturas públicas e edificações ${ }^{18}$. Destacam-se os distritos de Bonsucesso (40 óbitos) e Vieira (40 pessoas desaparecidas), localizados na zona rural de Teresópolis. Em Bonsucesso, os domicílios que foram mais atingidos estavam localizados junto às margens do rio. Foram identificadas também áreas de alta declividade, sem cobertura vegetal, potencializando os desastres ocorridos. De maneira geral, os deslizamentos de terra ocorridos na região Serrana estavam associados minimamente com a ocupação antrópica ${ }^{14}$.

Segundo Freitas et al. ${ }^{19}$ o crescimento populacional acelerado dos municípios da região Serrana, aliado a sua distribuição desordenada pelo território, resultou em ocupações nas margens de rios e encostas. A inexistência de uma política habitacional capaz de dar conta das demandas por habitação digna e segura está na origem de grande parte dos desastres.

Para analisar o impacto da mortalidade na região Serrana do Rio de Janeiro, foram utilizados dados do SIM (Agrupamento X30-X39 - Exposição às forças da natureza), que comparados aos dados do CEPED, mostraram coerência, apesar das diferenças observadas, como mostra a Tabela 5.

\section{É possível observar um padrão de mortalidade para os municípios mais atingidos por desastres?}

Para responder a esta questão também foram utilizados os dados do SIM, principalmente porque possibilitam uma análise mais detalhada das características da população que foi a óbito nos desastres. Foram trabalhados apenas os dados referentes ao município de Nova Friburgo, por apresentar maior número de óbitos. Para sintetizar as informações, foram utilizados os três grandes grupos etários ( 0 a 14 anos - jovens; 15 a 59 anos - pessoas em idade ativa; 60 anos e mais - idosos), que têm como objetivo nos fornecer um panorama comparativo entre o padrão de mortalidade estabelecido em 2010 e a mortalidade ocorrida no ano do desastre, e somente a mortalidade ocasionada pelo desastre.

Inicialmente, para uma compreensão da composição etária do município de Nova Friburgo, observou-se que neste município, seguindo o padrão do Estado do Rio de Janeiro, apresenta a concentração da população no grupo etário de 15 a 59 anos $(65,57 \%$ em 2000 e 65,99\% em 2010), conforme Tabela 6. Em 10 anos, houve um aumento da proporção da população de 60 anos e mais (10,55\% em 2000 e 14,32\% em 2010), e diminuição da proporção de indivíduos com 0 a 14 anos (23,87\% em 2000 e 19,68\% em 2010). O município de Nova Friburgo possui população masculina maior que a população feminina nos

Tabela 5. Óbitos totais dos municípios de Teresópolis, Petrópolis e Nova Friburgo, em 2011, obtido pelo CEPED e pelo SIM, e a diferença comparativa entre as duas fontes de dados.

\begin{tabular}{lrrc}
\hline & SIM & CEPED & Diferença dos óbitos \\
\hline Teresópolis & 271 & 388 & 117 \\
Petrópolis & 45 & 71 & 26 \\
Nova Friburgo & 349 & 429 & 80
\end{tabular}

Fonte: CEPED e Sistema de Informação sobre Mortalidade (SIM/DATASUS) - Total do Agrupamento (X30-X39)

Exposição às forças da natureza. 
grupos etários de 0 a 14 anos, em 2000 e em 2010, e de 15 a 59 anos em 2010, e menor no grupo etário de 60 anos e mais.

Em relação a mortalidade, os óbitos ocorridos em 2000 e em 2010 apresentam as maiores proporções no grupo etário de 60 anos e mais (Tabela 7). Em Nova Friburgo, a população feminina é maior que a masculina neste grupo etário, e apresentou também que as maiores proporções de óbitos ocorrem entre as mulheres de 60 anos e mais (66,73\% em 2000 e 76,65\% em 2010), em relação aos óbitos masculinos (50,77\% em 2000 e $62,93 \%$ em 2010). Verificou-se também uma diminuição da proporção de óbitos totais, entre homens e mulheres, nos demais grupos etários (0 a 14 anos e 15 a 59 anos) em 2010.
Ao comparar os óbitos ocorridos em 2011 e os óbitos ocorridos no desastre no município de Nova Friburgo, a Tabela 8 mostra que, em 2011, o desastre teve um impacto determinante nas proporções de óbitos totais. Analisando apenas os óbitos decorrentes do desastre (retirados do SIM - Categoria X37 - Vítima de tempestade cataclísmica), o grupo de 15 a 59 anos apresentou maior proporção de óbitos (55,49\%), seguido do grupo etário de 0 a 14 anos (25\%) e 60 anos e mais $(19,51 \%)$. Esta ordem também foi observada entre óbitos masculinos e óbitos femininos.

Em Nova Friburgo foi possível observar que a intensidade do desastre aumentou o impacto da mortalidade em 2011, quando comparados a 2000 e 2010 (Tabela 8). O grupo etário de

Tabela 6. Percentuais da população total e por sexo do município de Nova Friburgo dos anos 2000 e 2010.

\begin{tabular}{lcccccccc}
\hline \multirow{2}{*}{ Grupos etários } & \multicolumn{2}{c}{ População total (\%) } & & \multicolumn{2}{c}{ População feminina (\%) } & & \multicolumn{2}{c}{ População masculina (\%) } \\
\cline { 2 - 3 } \cline { 7 - 8 } \cline { 7 - 8 } & $\mathbf{2 0 0 0}$ & $\mathbf{2 0 1 0}$ & & $\mathbf{2 0 0 0}$ & $\mathbf{2 0 1 0}$ & & $\mathbf{2 0 0 0}$ & $\mathbf{2 0 1 0}$ \\
\hline 0 a 14 anos & 23,87 & 19,68 & & 22,60 & 18,57 & & 25,22 & 20,89 \\
15 a 59 anos & 65,57 & 65,99 & & 65,82 & 65,73 & & 65,32 & 66,28 \\
60 anos e mais & 10,55 & 14,32 & & 11,58 & 15,70 & & 9,46 & 12,83 \\
\hline
\end{tabular}

Fonte: IBGE.

Tabela 7. Percentuais dos óbitos totais e por sexo do município de Nova Friburgo dos anos 2000 e 2010.

\begin{tabular}{|c|c|c|c|c|c|c|}
\hline \multirow{2}{*}{ Grupos etários } & \multicolumn{2}{|c|}{ Óbitos totais (\%) } & \multicolumn{2}{|c|}{ Óbitos mulheres (\%) } & \multicolumn{2}{|c|}{ Óbitos homens(\%) } \\
\hline & 2000 & 2010 & 2000 & 2010 & 2000 & 2010 \\
\hline 0 a 14 anos & 5,03 & 2,69 & 5,98 & 1,77 & 4,36 & 3,40 \\
\hline 15 a 59 anos & 37,59 & 28,36 & 27,29 & 21,58 & 44,87 & 33,67 \\
\hline 60 anos e mais & 57,38 & 68,95 & 66,73 & 76,65 & 50,77 & 62,93 \\
\hline
\end{tabular}

Fonte: Sistema de Informação sobre Mortalidade (SIM/DATASUS).

Tabela 8. Percentuais dos óbitos totais e por sexo do município de Nova Friburgo em 2011 e total de óbitos decorrentes do desastre de 2011.

\begin{tabular}{|c|c|c|c|c|c|c|}
\hline \multirow{2}{*}{ Grupos etários } & \multicolumn{2}{|c|}{ Óbitos totais (\%) } & \multicolumn{2}{|c|}{ Óbitos mulheres (\%) } & \multicolumn{2}{|c|}{ Óbitos homens(\%) } \\
\hline & 2011 & desastre $(2010)^{*}$ & 2011 & desastre (2011) & 2011 & desastre (2011) \\
\hline 0 a 14 anos & 7,13 & 25,00 & 7,09 & 21,02 & 7,16 & 28,65 \\
\hline 15 a 59 anos & 34,55 & 55,49 & 28,86 & 57,96 & 39,37 & 53,22 \\
\hline 60 anos e mais & 58,32 & 19,51 & 64,05 & 21,02 & 53,47 & 18,13 \\
\hline
\end{tabular}

* Total da categoria X37 (Vítima de tempestade cataclísmica). 
0 a 14 anos apresentou proporção de 7,13\% dos óbitos totais, valor maior que os encontrados anteriormente (5,03\% em 2000 e 2,69\% em 2010). Destacam-se neste grupo etário, os óbitos femininos, que em 2011 apresentaram proporção de 7,09\%, superando os anos de 2000 (5,98\%) e 2010 (1,77\%). Já o grupo de 15 a 59 anos, em 2011 apresentou aumento em relação ao ano de 2010, mas não superou as proporções dos óbitos ocorridos em 2000. E o grupo etário de 60 anos e mais apresentou uma diminuição da proporção de óbitos em 2011 (58,32\%), em relação a 2010 (68,95\%).

A Figura 2 mostra a proporção de óbitos por grupos quinquenais de idade, possibilitando a observação de um padrão semelhante entre os anos de 2000, 2010 e 2011: queda da proporção de óbitos no grupo etário de 5 a 9 anos, seguida de um aumento contínuo a partir dos 15 anos. O padrão diferenciado das proporções de óbitos decorrentes do desastre em 2011, apresentou valores expressivos nos grupos etários de 5 a 9 anos e 25 a 29 anos, e valores baixos a partir do grupo etário de 70 a 74 anos. Esta análise permite apontar maior proporção de óbitos entre crianças e adultos de 25 a 39 anos.

O momento que os escorregamentos atingiram o município de Nova Friburgo pode ter influenciado nos padrões de mortalidade por idade e sexo, pois o risco de ser atingido pode estar relacionado com a hora do dia que influenciam o lugar onde as pessoas se encontram. O horário que Nova Friburgo foi atingida consta por volta das 4 horas da manhã $\tilde{a}^{5}$, sugerindo que as pessoas estavam em seus domicílios na hora do desastre, com capacidade de fuga diminuída por se encontrar dormindo.

Entre as mulheres (Figura 3a), as maiores proporções de óbitos em 2011 encontram-se nas idades mais avançadas, o que seria de se esperar em um contexto no qual a mortalidade infantil diminui de maneira significativa a sua relevância. Este fato também é observado entre os homens (Figura 3b). Já no desastre, as maiores proporções estão entre os grupos etários de 5 a 9 anos, 20 a 24 anos e 55 a 59 anos, entre as mulheres. Já entre os homens, as maiores proporções de óbitos decorrentes dos desastres estão entre os grupos de 5 a 9 anos, 10 a 14 anos, 30 a 34 anos e 40 a 44 anos. As Figuras $3 \mathrm{a}$ e $3 \mathrm{~b}$ mostram que o desastre impacta de diferentes formas os diversos grupos etários em ambos os sexos.

O que esses dados indicam é que grupos específicos, principalmente crianças, podem ser os mais atingidos em situações de desastre. Existem variações também em termos da distribuição dos óbitos por sexo em faixas etárias específicas. Entretanto, para obter informações mais conclusivas seria necessário ampliar o escopo da análise, com o estudo detalhado de maior número de casos.

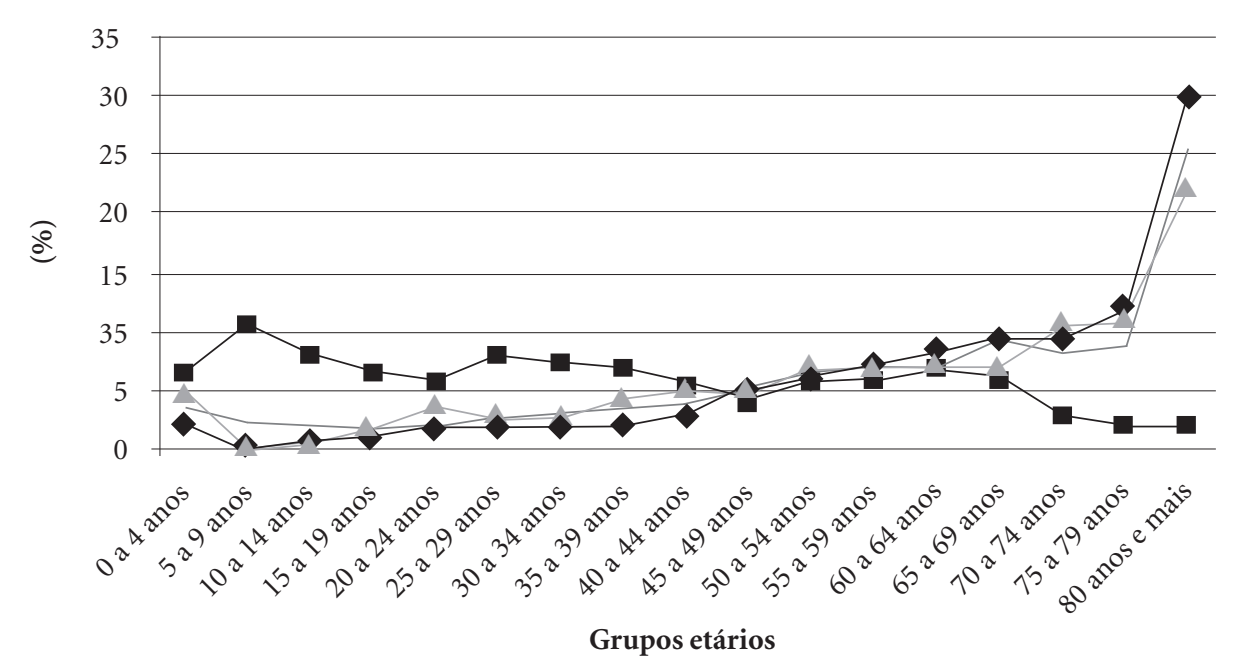

$\begin{array}{lll}\text { — proporção óbitos no desastre } 2011 & \text { proporção óbitos } 2000 \\ \text { proporção óbitos } 2011 & -\quad \text { proporção óbitos } 2010\end{array}$

Figura 2. Proporção de óbitos do município de Nova Friburgo em 2000, 2010 e 2011 e dos óbitos decorrentes do desastre em 2011, segundo grupos etários. Fonte: Sistema de Informação sobre Mortalidade (SIM/DATASUS). 
(a)

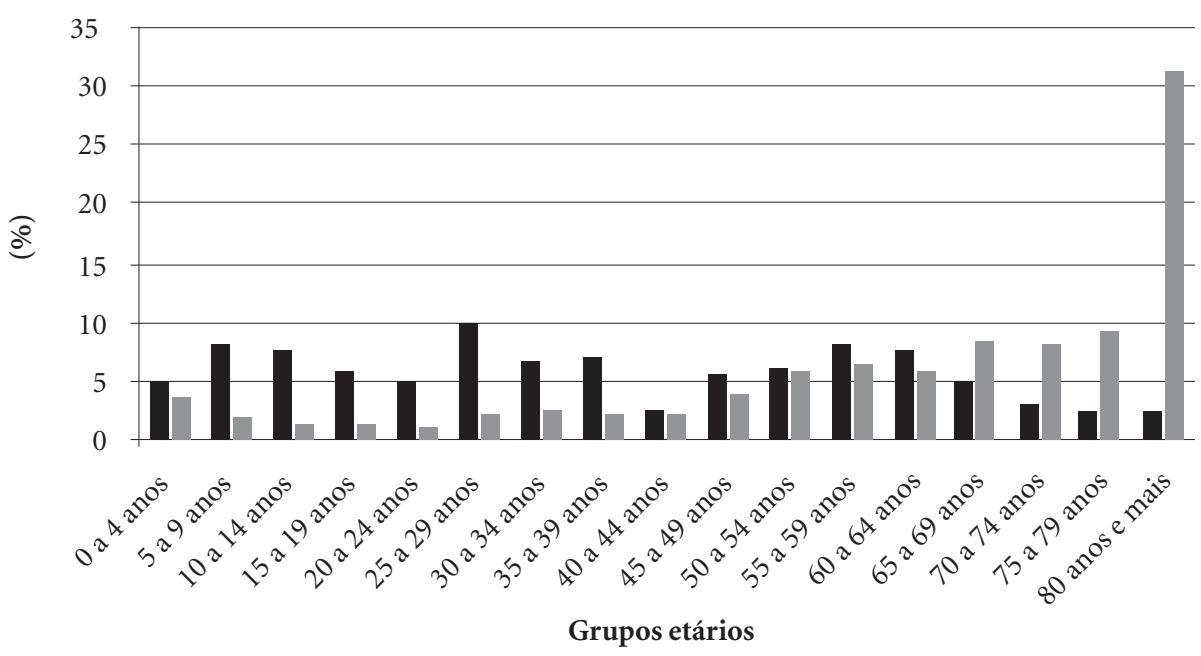

proporção óbitos femininos no desastre* 2011

proporção óbitos femininos 2011

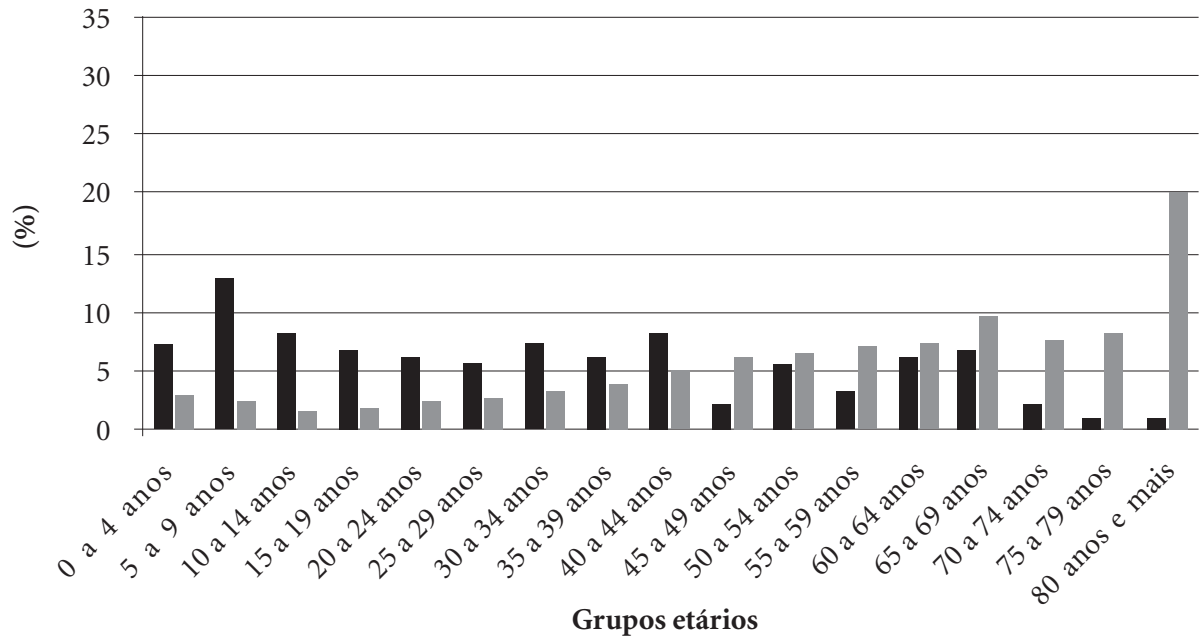

(b)

proporção óbitos masculinos no desastre* 2011

proporção óbitos masculinos 2011

Figura 3. Proporção de óbitos das mulheres (a) e dos homens (b) do município de Nova Friburgo em 2011 e dos óbitos decorrentes do desastre em 2011.

*Total da categoria X37 (Vítima de tempestade cataclísmica).

Fonte: Sistema de Informação sobre Mortalidade (SIM/DATASUS).

\section{Considerações Finais}

A disponibilização de informações sobre as decorrências resultantes dos desastres são impor- tantes para a atuação dos governos e demais instituições, delineando ações de prevenção e potencializando as situações de enfrentamento do pós-desastre. Por conta desta perspectiva, foram 
analisadas as principais bases de dados disponíveis sobre desastres no Brasil.

O EM-DAT, marcado por dificuldades materializadas na variação de conceitos e metodologias de obtenção de dados de diferentes países, consiste em uma base internacional de dados relacionados a desastres de fácil acesso e disponibilidade dos dados. Foram identificados inconsistências em termos de omissão, quantificação e classificação dos desastres, quando os dados foram comparados com a base de dados do Atlas Brasileiro de Desastres Naturais, sistematizado pelo $\mathrm{CEPED}^{10}$, que apresentou maior cobertura no número de registro de desastres e maior detalhamento dos eventos ocorridos.

O Anuário de Desastres Naturais no Brasil, publicado pelo Ministério da Integração Nacional, em sua 2a versão (lançados em 2011 e 2012), representa um marco para a sistematização de dados e também um avanço, caso seja mantido no longo prazo, para a análise longitudinal e comparativa dos desastres que ocorrem em todo o território nacional. Ao comparar os dados do Anuário com os dados do SIM, foi observado que a diferença dos números absolutos de óbitos foi pequena. Utilizados para a análise de mortalidade do megadesastre do Rio de Janeiro, os dados do SIM forneceram informações detalhadas, como idade e sexo dos indivíduos que morreram decorrentes do desastre, trazendo a possibilidade de identificar a possível existência de um padrão de mortalidade.

Com essa análise das informações foi possível comprovar a hipótese inicial de que cada uma das bases de dados apresenta resultados específicos, com acuidade diferenciada. E isso aponta para a necessidade de integração entre as diversas fontes, de maneira a possibilitar a disponibilização de informações que são fidedignas e que sirvam como subsídio para a construção de políticas públicas para esta questão que tende a ser cada vez mais relevante.

A hipótese de que as bases de dados deveriam evidenciar que a mortalidade por causas relacionadas a desastres está aumentando não pôde ser confirmada, tendo em vista que houve uma grande oscilação na frequência e na gravidade dos desastres ao longo do tempo.

A partir dos dados analisados, o Estado do Rio de Janeiro se destaca pelo seu histórico relacionado aos desastres, totalizando 22,98\% da população total do Rio de Janeiro afetada por desastres, com 854 óbitos, no período de 19912010. Apenas em 2011, com o megadesastre, foram totalizados 858 óbitos, segundo o SIM, número acentuado quando comparado à média em 15 anos (1996-2010), de 5 óbitos no Estado.

O megadesastre da Região Serrana do Rio de Janeiro foi considerado catastrófico na história do país, situação verificada nos três municípios mais afetados: Nova Friburgo, Petrópolis e Teresópolis. No município de Nova Friburgo, a mortalidade apresentou características diferenciadas, resultantes do impacto incisivo do desastre, que influenciou as taxas de mortalidade do município em 2011. O grupo etário de 5 a 9 anos, foi o mais atingido entre homens e mulheres, além de grupos etários de 20 a 24 anos (para mulheres) e 30 a 34 (para homens). Os resultados mostraram que o desastre impactou de diferentes formas os diversos grupos etários em ambos os sexos, principalmente as crianças no caso de Nova Friburgo.

Esses dados permitiram corroborar a hipótese de que a mortalidade por desastres é diferencial por sexo e por idade, sendo que a composição etária de uma população pode influenciar na vulnerabilidade aos desastres.

A ocupação dos diferentes espaços dentro da área urbana decorre de um processo social, marcado por uma distribuição desigual de inserção no ganho com as atividades econômicas. Os espaços reservados para a população de baixa renda são, em grande parte, expostos a perigos de deslizamentos de encostas e de inundações. Esses perigos se transformam em riscos iminentes, principalmente em função dos eventos climáticos extremos, que potencializam as situações de desastre. Entender como a cidade se configura dessa forma é fundamental para compreender os desastres como construção social. E trabalhar de maneira correta as informações é fundamental para essa compreensão, e também para gerar políticas que sejam capazes de evitar novos desastres.

\section{Colaboradores}

RL Carmo e TM Anazawa participaram igualmente de todas as etapas de elaboração do artigo. 


\section{Referências}

1. Intergovernmental Panel On Climate Change (IPCC). Climate Change 2007: The Physical Science Basis, Summary for Policymakers. 2007 [acessado 2013 ago 19]. Disponível em: http://www.ipcc.ch/SPM2feb07.pdf

2. Dynes RR, Drabek TE. The structure of disaster research: its policy and disciplinary implications. International Journal of Mass Emergencies and Disasters 1994; 12(1):5-23.

3. Quarantelli EL, Dynes RR. Response to social crisis and disaster. Annual Review of Sociology 1977; (3):23-49.

4. Chesnais JC. The demographic transition: Stages, patterns, and economic implications. Clarendon Press: Oxford; 1992.

5. Lee RD, Reher DS. Introduction: The landscape of demographic transition and its aftermath. Population and Development Review 2011; 37(Supl. 1):1-7.

6. Carmo RL, Dagnino RS, Johansen IC. Transição demográfica e transição do consumo urbano de água no Brasil. Rev Brasileira de Estudos de População. 2014; 31(1):169-190.

7. Carmo RL. Urbanização e desastres: desafios para a segurança humana no Brasil. In: Carmo RL, Valencio $\mathrm{N}$, organizadores. Segurança Humana em contextos de desastres. São Carlos: Editora Rima; 2014. p. 1-14.

8. Emergency Database (EM-DAT). OFDA/CRED The Office of Foreign Disaster Assistance/Centre for Research on the Epidemiology of Disasters - Université Catholique de Louvain, Brussels, Belgium. (s/d.). [acessado 2013 set 16] Disponível em: <http://www.emdat. be/natural-disasters-trends $>$.

9. Brasil. Ministério da Integração Nacional. Anuário Brasileiro de Desastres Naturais 2011. Brasília: Centro Nacional de Gerenciamento de Riscos e Desastres (CENAD); 2012.

10. Centro Universitário de Estudos e Pesquisas sobre Desastres (CEPED). Atlas Brasileiro de Desastres Naturais 1991 a 2010: volume Brasil. Florianópolis: UFSC; 2012.

11. Marcelino EV, Nunes LH, Kobiyama M. Banco de dados de desastres naturais: análise de dados globais e regionais. Caminhos da Geografia 2006; 6(19):130-149.
12. Departamento de Informática do SUS (DATASUS). SIM - Sistema de Informações sobre Mortalidade. (s/d). [acessado $2013 \mathrm{dez} 20]$. Disponível em: http://tabnet. datasus.gov.br/cgi/sim/obt10descr.htm.

13. Departamento de Informática do SUS (DATASUS). $10^{a}$ Revisão da Classificação Internacional de Doenças CID-10. 2008 [acessado 2013 dez 20]. Disponível em: http://www.datasus.gov.br/cid10/V2008/cid10.htm.

14. Brasil. Ministério da Integração Nacional. Anuário Brasileiro de Desastres Naturais 2012. Brasília: Centro Nacional de Gerenciamento de Riscos e Desastres (CENAD); 2013.

15. Centro Universitário de Estudos e Pesquisas sobre Desastres (CEPED). Diretrizes em Redução de Riscos de Desastres Região Serrana do Rio de Janeiro. Florianópolis: CEPED-UFSC; 2011.

16. Instituto Brasileiro de Geografia e Estatística (IBGE). Censo Demográfico 2010. Rio de Janeiro: IBGE; 2010.

17. Dourado F, Arraes TC, Silva MF. O Megadesastre da Região Serrana do Rio de Janeiro - as Causas do Evento, os Mecanismos dos Movimentos de Massa e a Distribuição Espacial dos Investimentos de Reconstrução no Pós-Desastre. Anuário do Instituto de Geociências UFRJ 2012; 35(2):43-54.

18. Brasil. Ministério do Meio Ambiente. Relatório de Inspeção Área atingida pela tragédia das chuvas Região Serrana do Rio de Janeiro. Brasília: Secretaria de Biodiversidade e Florestas; 2011.

19. Freitas CM, Carvalho ML, Ximenes EF, Arraes EF, Gomes JO. Vulnerabilidade socioambiental, redução de riscos de desastres e construção da resiliência: lições do terremoto no Haiti e das chuvas fortes na Região Serrana, Brasil. Cien Saude Colet 2012; 17(6):1577-1586.

Artigo apresentado em 10/03/2014

Aprovado em 10/06/2014

Versão final apresentada em 12/06/2014 